\title{
An Adaptive Total Generalized Variation Model with Augmented Lagrangian Method for Image Denoising
}

\author{
Chuan He, ${ }^{1}$ Changhua Hu, ${ }^{1}$ Xiaogang Yang, ${ }^{2}$ Huafeng He, ${ }^{1}$ and Qi Zhang ${ }^{1}$ \\ ${ }^{1}$ Unit 302, Xian Institute of High-Tech, Xi'an 710025, China \\ ${ }^{2}$ Unit 303, Xian Institute of High-Tech, Xi'an 710025, China \\ Correspondence should be addressed to Chuan He; hechuan8512@163.com
}

Received 3 March 2014; Revised 20 May 2014; Accepted 25 May 2014; Published 10 July 2014

Academic Editor: Fatih Yaman

Copyright (c) 2014 Chuan He et al. This is an open access article distributed under the Creative Commons Attribution License, which permits unrestricted use, distribution, and reproduction in any medium, provided the original work is properly cited.

\begin{abstract}
We propose an adaptive total generalized variation (TGV) based model, aiming at achieving a balance between edge preservation and region smoothness for image denoising. The variable splitting (VS) and the classical augmented Lagrangian method (ALM) are used to solve the proposed model. With the proposed adaptive model and ALM, the regularization parameter, which balances the data fidelity and the regularizer, is refreshed with a closed form in each iterate, and the image denoising can be accomplished without manual interference. Numerical results indicate that our method is effective in staircasing effect suppression and holds superiority over some other state-of-the-art methods both in quantitative and in qualitative assessment.
\end{abstract}

\section{Introduction}

In the past few decades, many variation or partial differential equation (PDE) based restoration models [1-7] have been proposed to recover images from degraded observations, due to the ability of preserving significant image features such as edges or textures. Among these models, the total variation (TV) model, also named the Rudin-Osher-Fatemi (ROF) model [1], is distinguished for excellent edge preserving ability and becomes one of the most widely used regularizers in image restoration $[1,2,8-10]$. In particular, the TV denoising problem is in the following form:

$$
\underset{u}{\operatorname{argmin}} \int_{\Omega}|\nabla u| \mathrm{d} \mathbf{x}+\frac{\lambda}{2} \int_{\Omega}\left|u-u_{0}\right|^{2} \mathrm{~d} \mathbf{x}
$$

where $\Omega$ is an open bounded domain in two dimensions, $u$ is the image to be restored, $u_{0}$ is the observation containing Gaussian white noise, and $\lambda$ is the regularization parameter which balances the regularization term and the data fidelity term. $\int_{\Omega}|\nabla u| \mathrm{d} \mathbf{x}$ is the TV seminorm of the bounded variation (BV) space $\mathrm{BV}(\Omega)$. The TV model is highly effective in preserving edges and corners, compared with the quadratic Tikhonov model. However, only when the original image is piecewise constant, the TV model is proved to be optimal. In fact, staircasing effect usually appears because most of natural images are not piecewise constant. Staircasing effect cannot meet the demands of human vision, due to the new artificial edges which do not exist in original images.

To overcome the drawback of the TV model, researchers suggest introducing the higher-order derivatives of image functions $[3-7,12-16]$. In order to eliminate the staircasing effect of TV model, Chambolle and Lions [14] proposed the following infimal-convolution minimization functional:

$$
\begin{gathered}
\underset{u, v}{\operatorname{argmin}} \int_{\Omega}|\nabla u-\nabla v| \mathrm{d} \mathbf{x}+\alpha \int_{\Omega}\left|\partial^{2} v\right| \mathrm{d} \mathbf{x} \\
+\lambda \int_{\Omega}\left|u-u_{0}\right|^{2} \mathrm{~d} \mathbf{x},
\end{gathered}
$$

where discontinuous components of the image are allotted to $u-v$ while regions of moderate slopes are assigned to $v$. The above model was proved to be practically efficient. Later, a modified form of (2) was proposed in [5] and its regularizer is of the following form:

$$
\underset{u, v}{\operatorname{argmin}} \int_{\Omega}|\nabla u-\nabla v| \mathrm{d} \mathbf{x}+\alpha \int_{\Omega}|\Delta v| \mathrm{d} \mathbf{x}
$$


That is, the second-order derivative in (2) is substituted by the Laplacian in (3). The similar use of Laplacian operator can also be seen in some PDE-based methods [3].

Since the classical TV model could not distinguish jumps from smooth transitions, Chan et al. [12] considered an additional penalization of the discontinuities in images. Precisely, they adopt

$$
\int_{\Omega}|\nabla u| \mathrm{d} \mathbf{x}+\alpha \int_{\Omega} \psi(|\nabla u|) h(\Delta u) \mathrm{d} \mathbf{x}
$$

as the regularization term, where $\psi$ is a real-valued function whose value approaches 0 while $|\nabla u|$ approaches infinity. The absence of the staircasing effect for this choice was verified in [15].

Bredies et al. [17] proposed the concept of total generalized variation (TGV), which is considered to be the generalization of TV. The TGV model is defined as

$$
\begin{array}{r}
\operatorname{TGV}_{\boldsymbol{\alpha}}^{k}(u)=\sup \left\{\int_{\Omega} u \operatorname{div}^{k} v \operatorname{dx} \mid v \in C_{c}^{k}\left(\Omega, \operatorname{Sym}^{k}\left(\mathbb{R}^{d}\right)\right),\right. \\
\left\|\operatorname{div}^{l} v\right\|_{\infty} \leq \alpha_{l}, \\
l=0, \ldots, k-1\}
\end{array}
$$

with

$$
\begin{aligned}
& \operatorname{Sym}^{k}\left(\mathbb{R}^{d}\right)=\left\{\zeta: \frac{\mathbb{R}^{d} \times \mathbb{R}^{d} \cdots \times \mathbb{R}^{d}}{k \text { times }} \rightarrow \mathbb{R}\right. \\
&\mid \zeta \text { is } k \text {-linear and symmetric }\},
\end{aligned}
$$

where $d \geq 1$ denotes the image dimension, and, throughout this paper, we assume $d=2 ; \operatorname{Sym}^{k}\left(\mathbb{R}^{d}\right)$ is the space of symmetric $k$-tensors on $\mathbb{R}^{d} ; C_{c}^{k}\left(\Omega, \operatorname{Sym}^{k}\left(\mathbb{R}^{d}\right)\right)$ is the space of compactly supported symmetric tensor field; $\alpha_{l}$ is fixed positive parameter. From the definition of $\mathrm{TGV}_{\boldsymbol{\alpha}}^{k}$, we learn that it involves the derivatives of $u$ of order one to $k$. When $k=1$ and $\alpha_{0}=1, \mathrm{TGV}_{\boldsymbol{\alpha}}^{k}$ degenerates to the classical TV. Thus $\mathrm{TGV}$ can be seen as a generalization of TV.

TGV involves and balances higher-order derivatives of $u$. Image reconstruction with TGV regularization usually leads to result with piecewise polynomial intensities and sharp edges. Therefore, TGV can effectively suppress the staircasing effect. In [17], an accelerated first-order method of Nesterov [18] was proposed to solve the TGV-regularized denoising problem.

In this paper, we propose an adaptive second-order TGVregularized model for denoising and derive an augmented Lagrangian approach to handle the suggested model. Our denoising model is as follows:

$$
\underset{u}{\operatorname{argminTG}} \mathrm{TG}_{\boldsymbol{\alpha}}^{2}(u) \quad \text { s.t. } \quad \int_{\Omega}\left|u-u_{0}\right|^{2} \mathrm{~d} \mathbf{x} \leq c .
$$

According to the standard Lagrange duality, for a given $c$, there exists a nonnegative $\lambda$ such that

$$
\underset{u}{\operatorname{argmin}} \operatorname{TGV}_{\boldsymbol{\alpha}}^{2}(u)+\frac{\lambda}{2} \int_{\Omega}\left|u-u_{0}\right|^{2} \mathrm{~d} \mathbf{x}
$$

is equivalent to (6). However, with (6), we can automatically estimate the regularization parameter $\lambda$. We first utilize an indicator function of the feasible set to transform problem (6) into an unconstrained one; then the variable splitting technique is applied to transform the resulting unconstrained problem into a problem with linear penalizing constraints; finally, the obtained constrained problem is solved by the alternating direction method of multipliers (ADMM) [1922], which is an instance of the classical ALM. The resulting image denoising algorithm is effective in staircasing effect suppression compared with some TV-based denoising methods, due to the second-order TGV regularizer. Besides, it achieves the adaptive estimation of the regularization parameter without inner iterative scheme. It is worth noting that the idea of this paper can be extended to TGV models with higher order than two. However, for simplicity, we only treat the second-order model and this is adequate for a large class of natural images.

Our method differs from the previous works on at least two aspects. On one hand, compared with [16], which adopted the accelerated first-order method of Nesterov [18] to handle the unconstrained TGV-based denoising problem (7), we apply ALM to the constrained TGV-based denoising problem (6) and achieve the automatic estimation of the regularization parameter $\lambda$. Our strategy avoids the extra cost on the manual selection of $\lambda$ by try-and-error. On the other hand, compared with the existing TV-based adaptive methods $[10,23,24]$, we propose a more complicated adaptive method based on TGV, and it is apt to achieve more attractive results than the TV-based methods.

The outline of the rest of the paper is organized as follows. Section 2 provides the description of the adaptive second-order TGV-based model for image denoising. Based on the Lagrange duality, an equivalent form of $\mathrm{TGV}_{\alpha}^{2}$ is suggested. The derivation of the proposed method is presented in Section 3. Section 4 gives the numerical results that demonstrate the effectiveness of the proposed method. At last, Section 5 ends this paper with a brief conclusion.

\section{Adaptive Second-Order TGV-Based Model for Image Denoising}

The space of bounded generalized variation (BGV) functions of order $k$ with weight $\boldsymbol{\alpha}$ is defined as

$$
\mathrm{BGV}_{\alpha}^{k}(\Omega)=\left\{u \in L^{1}(\Omega) \mid \operatorname{TGV}_{\alpha}^{k}(u)<\infty\right\} .
$$

Correspondingly, the BGV norm is defined as

$$
\|u\|_{\mathrm{BGV}_{\alpha}^{k}}=\|u\|_{1}+\mathrm{TGV}_{\boldsymbol{\alpha}}^{k}(u) .
$$

The TGV seminorm rather than the BGV norm is usually used as a regularizer. 
In this paper, we just take $k=2$ into consideration for simplicity. The second-order TGV can be written as

$$
\begin{array}{r}
\operatorname{TGV}_{\boldsymbol{\alpha}}^{2}(u)=\sup \left\{\int_{\Omega} u \operatorname{div}^{2} v \mathrm{~d} \mathbf{x} \mid v \in C_{c}^{2}\left(\Omega, \operatorname{Sym}^{2}\left(\mathbb{R}^{d}\right)\right),\right. \\
\left.\|v\|_{\infty} \leq \alpha_{0},\|\operatorname{div} v\|_{\infty} \leq \alpha_{1}\right\},
\end{array}
$$

where the divergences are defined as

$$
(\operatorname{div} v)_{i}=\sum_{j=1}^{d} \frac{\partial v_{i j}}{\partial x_{j}}, \quad 1 \leq i \leq d ; \quad \operatorname{div}^{2} v=\sum_{i, j=1}^{d} \frac{\partial^{2} v_{i j}}{\partial x_{i} \partial x_{j}}
$$

In fact, $\operatorname{Sym}^{2}\left(\mathbb{R}^{d}\right)$ is equivalent to the space of all symmetric $d \times d$ matrices. The infinite norms in (10) are given by

$$
\begin{aligned}
\|v\|_{\infty} & =\sup _{\mathbf{x} \in \Omega}\left(\sum_{i, j=1}^{d}\left|v_{i j}(\mathbf{x})\right|^{2}\right)^{1 / 2}, \\
\|\operatorname{div} v\|_{\infty} & =\sup _{\mathbf{x} \in \Omega}\left(\sum_{i=1}^{d}\left|(\operatorname{div} v)_{i}\right|^{2}\right)^{1 / 2} .
\end{aligned}
$$

For the convenience of the derivation of our algorithm, we apply the discrete form in the following and the tensors and vectors are denoted in bold type font. In order to make use of ADMM, we apply an equivalent definition of $\operatorname{TGV}_{\boldsymbol{\alpha}}^{2}[17,22]$ based on the Lagrange duality. With this definition, we have

$$
\mathrm{TGV}_{\boldsymbol{\alpha}}^{2}(\mathbf{u})=\min _{\mathbf{p}} \alpha_{0}\|\varepsilon(\mathbf{p})\|_{1}+\alpha_{1}\|\nabla \mathbf{u}-\mathbf{p}\|_{1},
$$

where $\mathbf{u} \in \mathbb{R}^{m n}$ denotes an $m \times n$ image, $\mathbf{p} \in \mathbb{R}^{m n} \times \mathbb{R}^{m n}$ belongs to the two-dimensional 1-tensor field, and $\varepsilon$ denotes the symmetrized derivative operator. Suppose that $u_{i, j}$ and $\mathbf{p}_{i, j}$ denote the $(i, j)$ th components of $\mathbf{u}$ and $\mathbf{p}$, respectively. Then we have $\mathbf{p}_{i, j}=\left[p_{i, j, 1}, p_{i, j, 2}\right] \in \operatorname{Sym}^{1}\left(\mathbb{R}^{2}\right)$ and the $(i, j)$ th component of $\varepsilon(\mathbf{p})$ is given by

$$
\begin{aligned}
\varepsilon(\mathbf{p})_{i, j}= & {\left[\begin{array}{ll}
\varepsilon(\mathbf{p})_{i, j, 1} & \varepsilon(\mathbf{p})_{i, j, 3} \\
\varepsilon(\mathbf{p})_{i, j, 3} & \varepsilon(\mathbf{p})_{i, j, 2}
\end{array}\right] } \\
= & {\left[\begin{array}{cc}
\frac{\nabla_{x_{1}} p_{i, j, 1}}{\left(\nabla_{x_{2}} p_{i, j, 1}+\nabla_{x_{1}} p_{i, j, 2}\right)} & \frac{\left(\nabla_{x_{2}} p_{i, j, 1}+\nabla_{x_{1}} p_{i, j, 2}\right)}{2}
\end{array}\right] } \\
\in \operatorname{Sym}^{2}\left(\mathbb{R}^{2}\right) & 0 \leq i \leq m, 0 \leq j \leq n,
\end{aligned}
$$

where $\nabla_{x_{1}}$ and $\nabla_{x_{2}}$ denote the difference operators in directions $x_{1}$ and $x_{2}$. According to the definition of operators $\nabla$ and $\varepsilon,(\nabla \mathbf{u})_{i, j}$ and $\varepsilon(\mathbf{p})_{i, j}$ are two-dimensional 1-tensor and symmetric 2-tensor, respectively. Besides, the \|\|$_{1}$ s of $\mathbf{p}$ and $\varepsilon(\mathbf{p})$ are defined as $\|\mathbf{p}\|_{1}=\sum_{i, j}^{m, n}\left|\mathbf{p}_{i, j}\right|=$ $\sum_{i, j}^{m, n} \sqrt{p_{i, j, 1}^{2}+p_{i, j, 2}^{2}}$ and $\|\varepsilon(\mathbf{p})\|_{1}=\sum_{i, j}^{m, n}\left|\varepsilon(\mathbf{p})_{i, j}\right|=$ $\sum_{i, j}^{m, n} \sqrt{\varepsilon(\mathbf{p})_{i, j, 1}^{2}+\varepsilon(\mathbf{p})_{i, j, 2}^{2}+2 \varepsilon(\mathbf{p})_{i, j, 3}^{2}}$, respectively. The deduction of (13) is given in the Appendix.

Then the constrained second-order TGV-regularized denoising problem (6) can be rewritten as

$$
\begin{array}{ll}
\underset{\mathbf{u}, \mathbf{p}}{\operatorname{argmin}} & \alpha_{0}\|\varepsilon(\mathbf{p})\|_{1}+\alpha_{1}\|\nabla \mathbf{u}-\mathbf{p}\|_{1} \\
\text { s.t. } & \left\|\mathbf{u}-\mathbf{u}_{0}\right\|_{2}^{2} \leq c .
\end{array}
$$

\section{Methodology}

3.1. The Augmented Lagrangian Model of Adaptive TGVBased Denoising. Problem (15) can be transformed into an unconstrained problem, with the following discontinuous objective functional:

$$
\underset{\mathbf{u}, \mathbf{p}}{\operatorname{argmin}} \alpha_{0}\|\varepsilon(\mathbf{p})\|_{1}+\alpha_{1}\|\nabla \mathbf{u}-\mathbf{p}\|_{1}+I_{\Phi}(\mathbf{u}),
$$

where $I_{\Phi}(\mathbf{u})$ is the indicator function of the feasible set defined by

$$
I_{\Phi}(\mathbf{u})= \begin{cases}0, & \text { if } \mathbf{u} \in \Phi \triangleq\left\{\mathbf{u}:\left\|\mathbf{u}-\mathbf{u}_{0}\right\|_{2}^{2} \leq c\right\} \\ +\infty & \text { otherwise. }\end{cases}
$$

Note that $\Phi$ is a closed Euclidean ball centered at $\mathbf{u}_{0}$ with radius $\sqrt{c}$.

The solution of problem (16) suffers from its nonlinearity and nondifferentiability. Referring to the variable splitting, we introduce three auxiliary variables to simplify the solution process of (16): a variable $\mathbf{w}$ for liberating $\mathbf{u}$ out from the constraint of the feasible set; a variable $\mathbf{y}$ and a variable $\mathbf{z}$ for liberating $\varepsilon(\mathbf{p})$ and $\nabla \mathbf{u}-\mathbf{p}$ out from the nondifferentiable 1norms, respectively. Then problem (16) can be transformed into the following equivalent constrained problem:

$$
\begin{array}{ll}
\underset{\mathbf{u}, \mathbf{p}}{\operatorname{argmin}} & \alpha_{0}\|\mathbf{y}\|_{1}+\alpha_{1}\|\mathbf{z}\|_{1}+I_{\Phi}(\mathbf{w}) \\
\text { s.t. } & \mathbf{u}=\mathbf{w}, \quad \varepsilon(\mathbf{p})=\mathbf{y}, \quad \nabla \mathbf{u}-\mathbf{p}=\mathbf{z} .
\end{array}
$$

In order to liberate $\mathbf{u}$ out from the feasible set constraint, we introduce auxiliary variable w. Similar operation can also be found in [10]. Without this operation, we should resort to an inner iterative scheme to update the regularization parameter.

The corresponding augmented Lagrangian functional of (18) is defined as

$$
\begin{aligned}
\mathscr{L}_{\mathscr{A}}(\mathbf{u}, \mathbf{p}, \mathbf{w}, \mathbf{y}, \mathbf{z} ; \boldsymbol{\mu}, \boldsymbol{\xi}, \boldsymbol{\eta}) \triangleq & I_{\Phi}(\mathbf{w})-\langle\boldsymbol{\mu}, \mathbf{w}-\mathbf{u}\rangle \\
& +\frac{\beta_{1}}{2}\|\mathbf{w}-\mathbf{u}\|_{2}^{2}+\alpha_{0}\|\mathbf{y}\|_{1} \\
& -\langle\boldsymbol{\xi}, \mathbf{y}-\varepsilon(\mathbf{p})\rangle
\end{aligned}
$$




$$
\begin{aligned}
& +\frac{\beta_{2}}{2}\|\mathbf{y}-\varepsilon(\mathbf{p})\|_{2}^{2}+\alpha_{1}\|\mathbf{z}\|_{1} \\
& -\langle\boldsymbol{\eta}, \mathbf{z}-\nabla \mathbf{u}+\mathbf{p}\rangle \\
& +\frac{\beta_{3}}{2}\|\mathbf{z}-\nabla \mathbf{u}+\mathbf{p}\|_{2}^{2},
\end{aligned}
$$

where $\boldsymbol{\mu}, \boldsymbol{\xi}$, and $\boldsymbol{\eta}$ are Lagrange multipliers and $\beta_{1}, \beta_{2}$, and $\beta_{3}$ are penalty parameters which should be positive. According to the classical ADMM, we should solve the following iterative scheme:

$$
\begin{aligned}
& \mathbf{u}^{k+1}=\underset{\mathbf{u}}{\operatorname{argmin}} \mathscr{L}_{\mathscr{A}}\left(\mathbf{u}, \mathbf{p}^{k}, \mathbf{w}^{k}, \mathbf{y}^{k}, \mathbf{z}^{k} ; \boldsymbol{\mu}^{k}, \boldsymbol{\xi}^{k}, \boldsymbol{\eta}^{k}\right), \\
& \mathbf{p}^{k+1}=\underset{\mathbf{p}}{\operatorname{argmin}} \mathscr{L}_{\mathscr{A}}\left(\mathbf{u}^{k+1}, \mathbf{p}, \mathbf{w}^{k}, \mathbf{y}^{k}, \mathbf{z}^{k} ; \boldsymbol{\mu}^{k}, \boldsymbol{\xi}^{k}, \boldsymbol{\eta}^{k}\right), \\
& \mathbf{w}^{k+1}=\underset{\mathbf{w}}{\operatorname{argmin}} \mathscr{L}_{\mathscr{A}}\left(\mathbf{u}^{k+1}, \mathbf{p}^{k+1}, \mathbf{w}, \mathbf{y}^{k}, \mathbf{z}^{k} ; \boldsymbol{\mu}^{k}, \boldsymbol{\xi}^{k}, \boldsymbol{\eta}^{k}\right), \\
& \mathbf{y}^{k+1}=\underset{\mathbf{y}}{\operatorname{argmin}} \mathscr{L}_{\mathscr{A}}\left(\mathbf{u}^{k+1}, \mathbf{p}^{k+1}, \mathbf{w}^{k+1}, \mathbf{y}, \mathbf{z}^{k} ; \boldsymbol{\mu}^{k}, \boldsymbol{\xi}^{k}, \boldsymbol{\eta}^{k}\right), \\
& \mathbf{z}^{k+1}=\underset{\mathbf{z}}{\operatorname{argmin}} \mathscr{L}_{\mathscr{A}}\left(\mathbf{u}^{k+1}, \mathbf{p}^{k+1}, \mathbf{w}^{k+1}, \mathbf{y}^{k+1}, \mathbf{z} ; \boldsymbol{\mu}^{k}, \boldsymbol{\xi}^{k}, \boldsymbol{\eta}^{k}\right), \\
& \boldsymbol{\mu}^{k+1}=\boldsymbol{\mu}^{k}-\beta_{1}\left(\mathbf{w}^{k+1}-\mathbf{u}^{k+1}\right), \\
& \xi^{k+1}=\xi^{k}-\beta_{2}\left(\mathbf{y}^{k+1}-\varepsilon\left(\mathbf{u}^{k+1}\right)\right), \\
& \boldsymbol{\eta}^{k+1}=\boldsymbol{\eta}^{k}-\beta_{3}\left(\mathbf{z}^{k+1}-\nabla \mathbf{u}^{k+1}+\mathbf{p}^{k+1}\right) \text {. }
\end{aligned}
$$

3.2. Solution of the Subproblems. With the auxiliary w, the $\mathbf{u}$ subproblem becomes quadratic and irrelevant to the constraint of the feasible set. It allows the following objective:

$$
\begin{aligned}
\mathbf{u}^{k+1}= & \underset{\mathbf{u}}{\operatorname{argmin}} \frac{\beta_{1}}{2}\left\|\mathbf{w}^{k}-\mathbf{u}-\frac{\boldsymbol{\mu}^{k}}{\beta_{1}}\right\|_{2}^{2} \\
& +\frac{\beta_{3}}{2}\left\|\mathbf{z}^{k}-\nabla \mathbf{u}+\mathbf{p}^{k}-\frac{\boldsymbol{\eta}^{k}}{\beta_{3}}\right\|_{2}^{2}
\end{aligned}
$$

The minimization problem (21) can be solved by the following equation:

$$
\begin{aligned}
\mathbf{u}^{k+1}=\left(\frac{\beta_{1}}{\beta_{3}} \mathbf{I}+\nabla^{T} \nabla\right)^{-1} & \left(\frac{\beta_{1}}{\beta_{3}}\left(\mathbf{w}^{k}-\frac{\boldsymbol{\mu}^{k}}{\beta_{1}}\right)\right. \\
& \left.+\nabla^{T}\left(\mathbf{z}^{k}+\mathbf{p}^{k}-\frac{\boldsymbol{\eta}^{k}}{\beta_{3}}\right)\right) .
\end{aligned}
$$

With the circulant boundary condition of images, we can solve (22) with several FFTs and IFFTs $[8,10]$.
Following the same way, the subproblem with respect to $\mathbf{p}$ is also quadratic and we have the objective functional as follows:

$$
\begin{aligned}
\mathbf{p}^{k+1}= & \underset{\mathbf{p}}{\operatorname{argmin}} \frac{\beta_{2}}{2}\left\|\mathbf{y}^{k}-\varepsilon(\mathbf{p})-\frac{\boldsymbol{\xi}^{k}}{\beta_{2}}\right\|_{2}^{2} \\
& +\frac{\beta_{3}}{2}\left\|\mathbf{z}^{k}-\nabla \mathbf{u}^{k+1}+\mathbf{p}-\frac{\boldsymbol{\eta}^{k}}{\beta_{3}}\right\|_{2}^{2}
\end{aligned}
$$

Then, for $\mathbf{p}_{1}^{k+1}$, we have

$$
\begin{aligned}
& \left(\beta_{2} \nabla_{x_{1}}^{T} \nabla_{x_{1}}+\frac{\beta_{2}}{2} \nabla_{x_{2}}^{T} \nabla_{x_{2}}+\beta_{3} \mathbf{I}\right) \mathbf{p}_{1}^{k+1} \\
& =\beta_{2}\left[\nabla_{x_{1}}^{T}\left(\mathbf{y}_{1}^{k}-\frac{\xi_{1}^{k}}{\beta_{2}}\right)+\nabla_{x_{2}}^{T}\left(\mathbf{y}_{3}^{k}-\frac{\xi_{3}^{k}}{\beta_{2}}-\frac{1}{2} \nabla_{x_{1}} \mathbf{p}_{2}^{k}\right)\right] \\
& \quad+\beta_{3}\left(\nabla_{x_{1}} \mathbf{u}^{k+1}+\frac{\boldsymbol{\eta}_{1}^{k}}{\beta_{3}}-\mathbf{z}_{1}^{k}\right),
\end{aligned}
$$

and for $\mathbf{p}_{2}^{k+1}$, we have

$$
\begin{aligned}
& \left(\beta_{2} \nabla_{x_{2}}^{T} \nabla_{x_{2}}+\frac{\beta_{2}}{2} \nabla_{x_{1}}^{T} \nabla_{x_{1}}+\beta_{3} \mathbf{I}\right) \mathbf{p}_{2}^{k+1} \\
& =\beta_{2}\left[\nabla_{x_{2}}^{T}\left(\mathbf{y}_{2}^{k}-\frac{\boldsymbol{\xi}_{2}^{k}}{\beta_{2}}\right)+\nabla_{x_{1}}^{T}\left(\mathbf{y}_{3}^{k}-\frac{\xi_{3}^{k}}{\beta_{2}}-\frac{1}{2} \nabla_{x_{2}} \mathbf{p}_{1}^{k+1}\right)\right] \\
& \quad+\beta_{3}\left(\nabla_{x_{2}} \mathbf{u}^{k+1}+\frac{\boldsymbol{\eta}_{2}^{k}}{\beta_{3}}-\mathbf{z}_{2}^{k}\right),
\end{aligned}
$$

where $\mathbf{p}_{1}$ and $\mathbf{p}_{2}$ are the combinations of $p_{i, j, 1}$ and $p_{i, j, 2}(0 \leq$ $i \leq m, 0 \leq j \leq n)$, respectively. Similar to the solution of (22), problems (24) and (25) can also be solved conveniently through several FFTs and IFFTs under the assumption of the circulant boundary condition.

The subproblem for $\mathbf{y}$ can be written as

$$
\mathbf{y}^{k+1}=\underset{\mathbf{y}}{\operatorname{argmin}}\|\mathbf{y}\|_{1}+\frac{\beta_{2}}{2 \alpha_{0}}\left\|\mathbf{y}-\varepsilon\left(\mathbf{p}^{k+1}\right)-\frac{\xi^{k}}{\beta_{2}}\right\|_{2}^{2} .
$$

Problem (26) can be solved component-wisely through the following 4-dimensional shrinkage operation:

$$
\begin{aligned}
& \mathbf{y}_{i, j}^{k+1}= \max \left\{\left\|\varepsilon\left(\mathbf{p}^{k+1}\right)_{i, j}+\frac{\boldsymbol{\xi}_{i, j}^{k}}{\beta_{2}}\right\|_{2}-\frac{\alpha_{0}}{\beta_{2}}, 0\right\} \\
& \times \frac{\varepsilon\left(\mathbf{p}^{k+1}\right)_{i, j}+\left(\xi_{i, j}^{k} / \beta_{2}\right)}{\left\|\varepsilon\left(\mathbf{p}^{k+1}\right)_{i, j}+\left(\boldsymbol{\xi}_{i, j}^{k} / \beta_{2}\right)\right\|_{2}} \\
& 0 \leq i \leq m, \quad 0 \leq j \leq n .
\end{aligned}
$$

The $\mathbf{z}$ subproblem is given by

$$
\mathbf{z}^{k+1}=\underset{\mathbf{z}}{\operatorname{argmin}}\|\mathbf{z}\|_{1}+\frac{\beta_{3}}{2 \alpha_{1}}\left\|\mathbf{z}-\nabla \mathbf{u}^{k+1}+\mathbf{p}^{k+1}-\frac{\boldsymbol{\eta}^{k}}{\beta_{3}}\right\|_{2}^{2},
$$




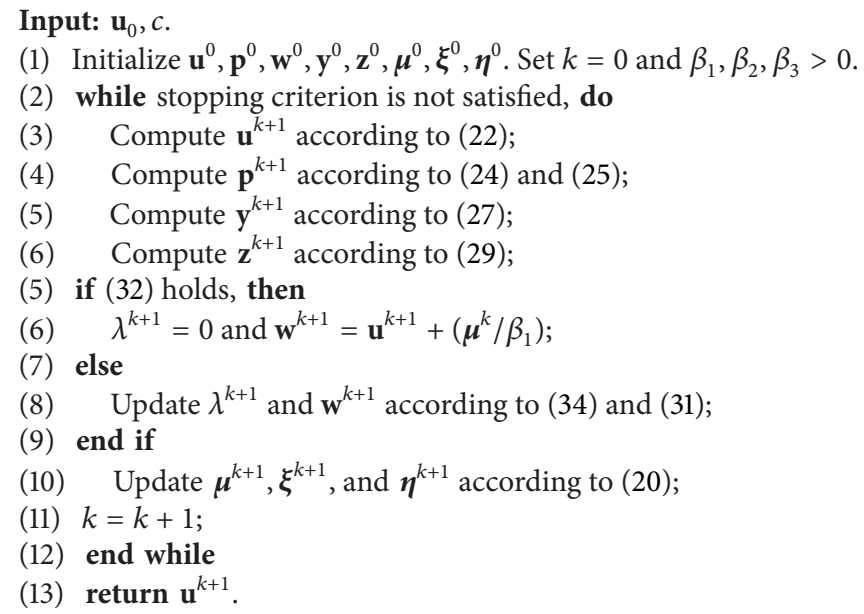

Algorithm 1: Algorithm TGV²ID-ADMM: Second-order TGV-regularized image denoising with ADMM.

and it can be solved component-wisely through the following 2-dimensional shrinkage operation:

$$
\begin{aligned}
& \mathbf{z}_{i, j}^{k+1}= \max \left\{\left\|\left(\nabla \mathbf{u}^{k+1}\right)_{i, j}+\frac{\boldsymbol{\eta}_{i, j}^{k}}{\beta_{3}}-\mathbf{p}_{i, j}^{k+1}\right\|_{2}-\frac{\alpha_{1}}{\beta_{3}}, 0\right\} \\
& \times \frac{\left(\nabla \mathbf{u}^{k+1}\right)_{i, j}+\left(\boldsymbol{\eta}_{i, j}^{k} / \beta_{3}\right)-\mathbf{p}_{i, j}^{k+1}}{\left\|\left(\nabla \mathbf{u}^{k+1}\right)_{i, j}+\left(\boldsymbol{\eta}_{i, j}^{k} / \beta_{3}\right)-\mathbf{p}_{i, j}^{k+1}\right\|_{2}} \\
& 0 \leq i \leq m, 0 \leq j \leq n .
\end{aligned}
$$

The subproblem with respect to $\mathbf{w}$ can be written as

$$
\begin{aligned}
\mathbf{w}^{k+1}= & \underset{\mathbf{w}}{\operatorname{argmin}} I_{\Phi}(\mathbf{w})+\frac{\beta_{1}}{2}\left\|\mathbf{w}-\left(\mathbf{u}^{k+1}+\frac{\boldsymbol{\mu}^{k}}{\beta_{1}}\right)\right\|_{2}^{2} \\
= & \underset{\mathbf{w}}{\operatorname{argmin}} \frac{\lambda^{k+1}}{2}\left\|\mathbf{w}-\mathbf{u}_{0}\right\|_{2}^{2} \\
& +\frac{\beta_{1}}{2}\left\|\mathbf{w}-\left(\mathbf{u}^{k+1}+\frac{\boldsymbol{\mu}^{k}}{\beta_{1}}\right)\right\|_{2}^{2} .
\end{aligned}
$$

Consequently, the solution of problem (30) is

$$
\mathbf{w}^{k+1}=\frac{\lambda^{k+1} \mathbf{u}_{0}+\beta_{1}\left(\mathbf{u}^{k+1}+\left(\boldsymbol{\mu}^{k} / \beta_{1}\right)\right)}{\lambda^{k+1}+\beta_{1}} .
$$

The solution of $\lambda^{k+1}$ falls into two cases according to the range of $\mathbf{u}^{k+1}+\left(\boldsymbol{\mu}^{k} / \beta_{1}\right)$. On one hand, if

$$
\left\|\mathbf{u}^{k+1}+\frac{\boldsymbol{\mu}^{k}}{\beta_{1}}-\mathbf{u}_{0}\right\|_{2}^{2} \leq c,
$$

we can set $\lambda^{k+1}=0$, and, obviously, $\mathbf{w}^{k+1}=\mathbf{u}^{k+1}+\boldsymbol{\mu}^{k} / \beta_{1}$ satisfies the feasible set constraint. On the other hand, if (32) is not true, $\mathbf{w}^{k+1}$ should fulfill the following equation:

$$
\left\|\mathbf{w}^{k+1}-\mathbf{u}_{0}\right\|_{2}^{2}=c .
$$

Substituting (31) into (33), we get

$$
\lambda^{k+1}=\frac{\beta_{1}\left\|\mathbf{u}^{k+1}+\left(\boldsymbol{\mu}^{k} / \beta_{1}\right)-\mathbf{u}_{0}\right\|_{2}}{\sqrt{c}}-\beta_{1} .
$$

The resulting image denoising algorithm is summarized in Algorithm 1 TGV²ID-ADMM.

The adoption of the variable $\mathbf{w}$ is essential for the adaptive estimate of the regularization parameter $\lambda$. With the assistance of $\mathbf{w}, \mathbf{u}$ is liberated out from the constraint of the feasible set. Thus, the update of $\lambda$ is free from the disturbance of the update of $\mathbf{u}$, and a closed form for updating $\lambda$ is achieved in each step without inner iteration. From functionals (16) and (18) we learn that, by setting $\left(\alpha_{0}, \alpha_{1}\right)=(0,1),(\mathbf{y}, \mathbf{p})=(\mathbf{0}, \mathbf{0})$, and $\beta_{2}=0$, Algorithm TGV ${ }^{2}$ ID-ADMM will degenerate to a TV-based denoising algorithm, and we denote this case as TGV ${ }^{1}$ ID-ADMM.

The convergence of Algorithm TGV ${ }^{2}$ ID-ADMM follows from the convergence analysis for the TV-based ADMM in $[11,25]$, due to the convex property of $\mathrm{TGV}_{\boldsymbol{\alpha}}^{2}$. In this paper, we do not repeat the lengthy analysis procedure. However, we have the following essential convergence theorem for the proposed method.

Theorem 1. For fixed $\beta_{1}, \beta_{2}, \beta_{3}>0$, the sequence $\left\{\mathbf{u}^{k}, \mathbf{p}^{k}, \mathbf{w}^{k}\right.$, $\left.\mathbf{y}^{k}, \mathbf{z}^{k}, \boldsymbol{\mu}^{k}, \boldsymbol{\xi}^{k}, \boldsymbol{\eta}^{k}, \lambda^{k}\right\}$ generated by Algorithm TGV $V^{2}$ ID-ADMM from any initial point $\left(\mathbf{u}^{0}, \mathbf{p}^{0}, \mathbf{w}^{0}, \mathbf{y}^{0}, \mathbf{z}^{0}, \boldsymbol{\mu}^{0}, \boldsymbol{\xi}^{0}, \boldsymbol{\eta}^{0}\right)$ converges to $\left(\mathbf{u}^{*}, \mathbf{p}^{*}, \mathbf{w}^{*}, \mathbf{y}^{*}, \mathbf{z}^{*}, \boldsymbol{\mu}^{*}, \boldsymbol{\xi}^{*}, \boldsymbol{\eta}^{*}, \lambda^{*}\right)$, where $\mathbf{u}^{*}$ is the solution of functional (15) and $\lambda^{*}$ is the regularization parameter corresponding to the feasible set constraint $\mathbf{u} \in \Phi$.

\section{Experiment Results}

In this section, we illustrate the effectiveness of the proposed algorithm on suppressing staircasing effect and removing Gaussian noise in image. Besides, we also show the robustness 
TABLE 1: Results of the staircasing effect reduction experiment.

\begin{tabular}{|c|c|c|c|c|}
\hline \multicolumn{5}{|c|}{ Piecewise affine image denoising with $\sigma=15$} \\
\hline Model & RMSE & PSNR & Iterations & Time $(\mathrm{s})$ \\
\hline Noised & 14.99 & 24.61 & - & - \\
\hline $\mathrm{TGV}^{2}$ & 1.89 & 42.61 & 155 & 18.21 \\
\hline $\mathrm{TGV}^{1}$ & 2.40 & 39.81 & 128 & 7.76 \\
\hline
\end{tabular}

of the results with respect to the penalty parameters. We performed our algorithm under MATLAB v7.8.0 and Windows 7 on a PC with an Intel Core (TM) i5 CPU at $3.20 \mathrm{GHz}$ and $8 \mathrm{~GB}$ of RAM.

The root mean squared error (RMSE) and the peak signalto-noise ratio (PSNR) used in comparison are defined as

$$
\begin{aligned}
& \text { RMSE }=\frac{\left\|\mathbf{u}-\mathbf{u}_{\text {clean }}\right\|_{2}}{\sqrt{m n}}, \\
& \text { PSNR }=10 \log _{10}\left(\frac{255^{2} \cdot m n}{\left\|\mathbf{u}-\mathbf{u}_{\text {clean }}\right\|_{2}^{2}}\right),
\end{aligned}
$$

where $\mathbf{u}_{\text {clean }}$ is the original image that contains no noise. Besides, in subsections 4.1 and 4.2 , we set the penalty parameters as $\beta_{1}=10^{(\mathrm{BSNR} / 10-1)} \times \beta$ and $\beta_{2}=\beta_{3}=\beta=0.3$ for $\mathrm{TGV}^{2}$ ID-ADMM ( $\beta_{2}=0$ for TGV ${ }^{1}$ ID-ADMM) to achieve consistently promising result with fast speed, where BSNR is the blurred signal-to-noise ratio defined by BSNR $=10 \log _{10}$ $\left(\operatorname{var}\left(\mathbf{u}_{0}\right) / \sigma^{2}\right)\left(\operatorname{var}\left(\mathbf{u}_{0}\right)\right.$ denotes the variance of $\left.\mathbf{u}_{0}\right)$.

4.1. Staircasing Effect Reduction by the Proposed Method. We first compare Algorithm TGV ${ }^{2}$ ID-ADMM with Algorithm $\mathrm{TGV}^{1} \mathrm{ID}-\mathrm{ADMM}$ to illustrate the effectiveness of $\mathrm{TGV}_{\boldsymbol{\alpha}}^{2}$ model in staircasing effect reduction. We use $\left\|\mathbf{u}^{k+1}-\mathbf{u}^{k}\right\|_{2}^{2} /$ $\left\|\mathbf{u}^{k}\right\|_{2}^{2} \leq 10^{-6}$ as the stopping criteria for these two algorithms, where $\mathbf{u}^{k}$ denotes the restored result in the $k$ th iteration. For the second-order case, we set $\left(\alpha_{0}, \alpha_{1}\right)=(3,1)$, whereas for the one-order case, we set $\left(\alpha_{0}, \alpha_{1}\right)=(0,1)$.

In this experiment, we use a synthetic piecewise affine image shown in Figure 1 as the test image. The original image is contaminated by Gaussian noise of standard variance $\sigma=15$ at first. Then we imply $\mathrm{TGV}^{2} \mathrm{ID}-\mathrm{ADMM}$ and $\mathrm{TGV}^{1} \mathrm{ID}-\mathrm{ADMM}$ to remove the noise. Table 1 shows the results in terms of RMSE, PSNR, total iterations, and CPU time. The ground truth, noised, and restored images by the two algorithms are displayed in Figure 1. Furthermore, for better visualization, we additionally provide the threedimensional close-ups of the marked regions of the two restored images in Figure 1. From Table 1 we observe that $\mathrm{TGV}^{2}$ ID-ADMM does better than TGV ${ }^{1}$ ID-ADMM in terms of both RMSE and PSNR. Figure 1 shows that the denoised image of $\mathrm{TGV}^{2} \mathrm{ID}-\mathrm{ADMM}$ almost contains no artificial edges in affine regions. In contrast, the restored result of $\mathrm{TGV}^{1} \mathrm{ID}$ ADMM contains obvious staircasing effect in affine regions. The three-dimensional closed-ups vividly demonstrate this phenomenon. This illustrates that our TGV-based algorithm is effective in staircasing effect reduction.

Table 1 also shows that, to accomplish the denoising task, TGV ${ }^{2}$ ID-ADMM usually costs more CPU time than $\mathrm{TGV}^{1} \mathrm{ID}-\mathrm{ADMM}$, since $\mathrm{TGV}_{\boldsymbol{\alpha}}^{2}$ model involves much more calculation. However, the cost is worthy due to the impressive improvement on both quantitative and qualitative restoration quality. Figure 2 displays the evolutions of $\lambda$ s and PSNRs achieved by the two algorithms. It is learnt that, the regularization parameters of both converge to the optimal points at last, which guarantees the automatic implementation of the two algorithms.

4.2. Comparison in Accuracy. In this subsection, we compare $\mathrm{TGV}^{2} \mathrm{ID}-\mathrm{ADMM}$ with the other two famous adaptive TVbased denoising algorithms: Chambolle's projection algorithm [23] and Split Bregman algorithm [24], both possessing public online implementations at "http://www.ipol.im/". Two natural images, Lena and Peppers both of size $512 \times 512$ shown in Figure 3, are used for comparison. The parameter setting for $\mathrm{TGV}^{2} \mathrm{ID}-\mathrm{ADMM}$ is the same as that in the previous subsection. We obtain the test results of the two competitors through online experimental operation.

We add Gaussian noise of standard variances of 20,30, and 40 to Lena and Peppers to obtain the noised observations, respectively. Then we apply these three algorithms to restore the noisy images. Table 2 shows the comparison results in terms of RMSE and PSNR. The best result for each comparison item is highlighted in bold type font. Table 2 shows that $\mathrm{TGV}^{2} \mathrm{ID}-\mathrm{ADMM}$ holds superiority on both RMSE and PSNR for all the tested cases. Figure 4 displays the noised Lena under Gaussian noise of $\sigma=$ 30 and the restorations by the three algorithms, whereas Figure 5 exhibits the noised Peppers under Gaussian noise of $\sigma=40$ and the corresponding restorations. Figures 4 and 5 demonstrate that $\mathrm{TGV}^{2} \mathrm{ID}$-ADMM obtains results with better visual impression and efficiently suppresses the staircasing effect. In contrast, both TV-based Chambolle's projection algorithm and TV-based Split Bregman algorithm achieve results with obvious staircasing effect. Since we apply test images with different levels of noise, the robustness of our algorithm towards the noise level is verified to a certain extent.

4.3. Solution Robustness with Respect to the Penalty Parameters. Although the positive assumption of penalty parameters is sufficient for the convergence of ADMM, the results of 

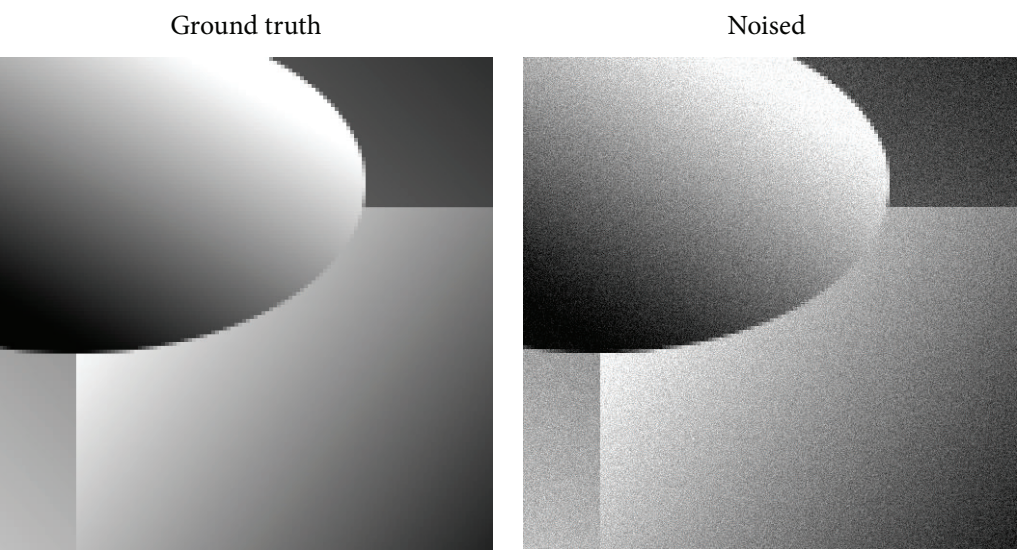

(a)
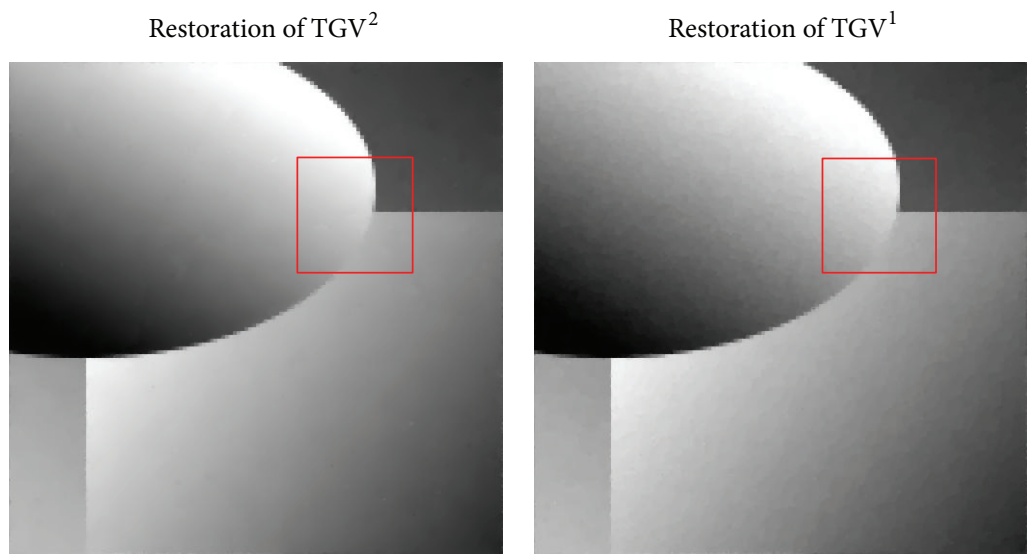

(b)
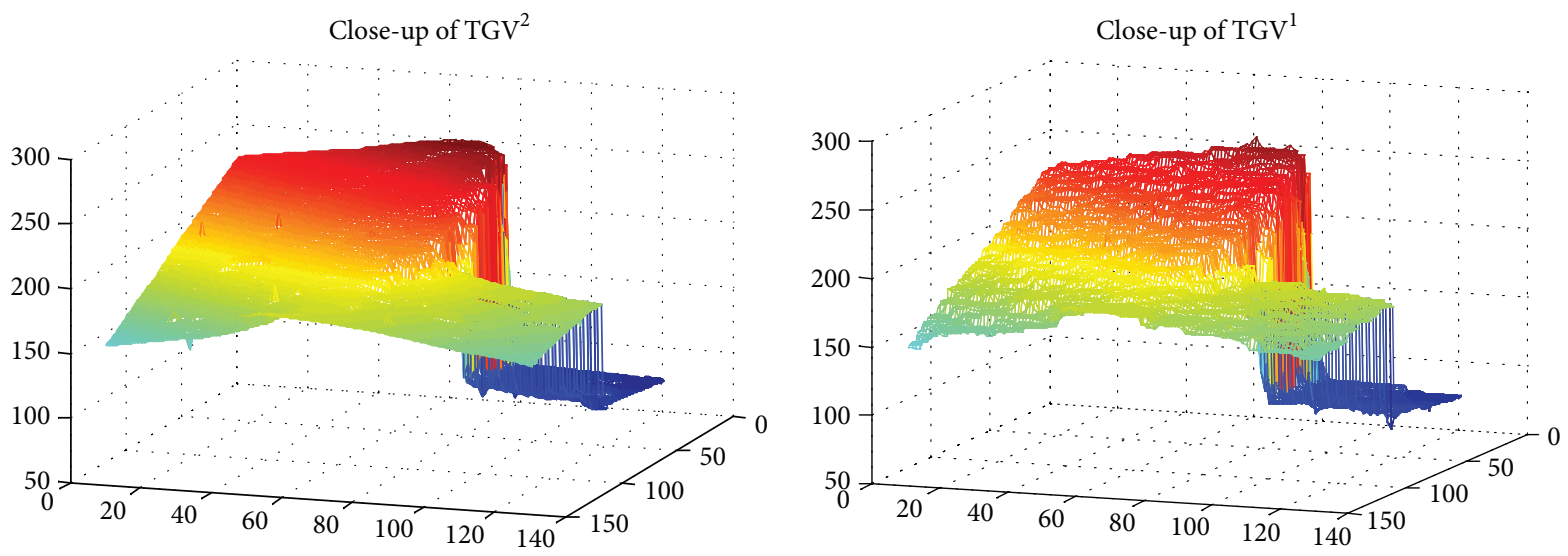

(c)

FIGURE 1: First row: ground truth and Gaussian noised $(\sigma=15)$ piecewise affine images; second row: restored images by TGV ${ }^{2}$ ID-ADMM and $\mathrm{TGV}^{1} \mathrm{ID}-\mathrm{ADMM}$; third row: three-dimensional close-ups of the marked regions in the two restored images.

ADMM are commonly influenced by the choice of the penalty parameters to a certain extent in practice. As suggested by a referee, we add an experiment to show the robustness of the results of $\mathrm{TGV}^{2} \mathrm{ID}-\mathrm{ADMM}$ with respect to the penalty parameters, under the two denoising background problems mentioned above, that is, the Lena denoising problem under
Gaussian noise of $\sigma=30$ and the Peppers denoising problem under Gaussian noise of $\sigma=40$. We still set $\beta_{1}=$ $10^{(\mathrm{BSNR} / 10-1)} \times \beta$ and $\beta_{2}=\beta_{3}=\beta$ but change $\beta$ from 0.01 to 1 with a step size of 0.01 . In Figure 6 , we plot PSNR versus $\beta$ for the denoised Lena and Peppers. Figure 6 demonstrates that the optimal $\beta$ should be focalized in $[0.2,0.3]$ and its 
TABLE 2: Comparison results in accuracy.

\begin{tabular}{|c|c|c|c|c|c|c|c|c|}
\hline \multirow{2}{*}{$\sigma$} & \multicolumn{2}{|c|}{ Noised } & \multicolumn{2}{|c|}{ TGV ${ }^{2}$ ID-ADMM } & \multicolumn{2}{|c|}{ Chambolle } & \multicolumn{2}{|c|}{ Split Bregman } \\
\hline & RMSE & PSNR & RMSE & PSNR & RMSE & PSNR & RMSE & PSNR \\
\hline \multicolumn{9}{|c|}{ Lena $512 \times 512$} \\
\hline 20 & 19.99 & 22.11 & 7.09 & 31.12 & 7.40 & 30.75 & 8.05 & 30.01 \\
\hline 30 & 30.00 & 18.59 & 8.67 & 29.37 & 9.45 & 28.62 & 9.95 & 28.17 \\
\hline 40 & 39.97 & 16.10 & 10.06 & 28.07 & 10.54 & 27.67 & 11.66 & 26.80 \\
\hline \multicolumn{9}{|c|}{ Peppers $512 \times 512$} \\
\hline 20 & 19.96 & 22.13 & 7.05 & 31.17 & 7.38 & 30.77 & 7.82 & 30.27 \\
\hline 30 & 29.98 & 18.59 & 8.68 & 29.36 & 9.43 & 28.64 & 9.92 & 28.20 \\
\hline 40 & 40.10 & 16.07 & 9.74 & 28.36 & 10.74 & 27.51 & 12.38 & 26.28 \\
\hline
\end{tabular}

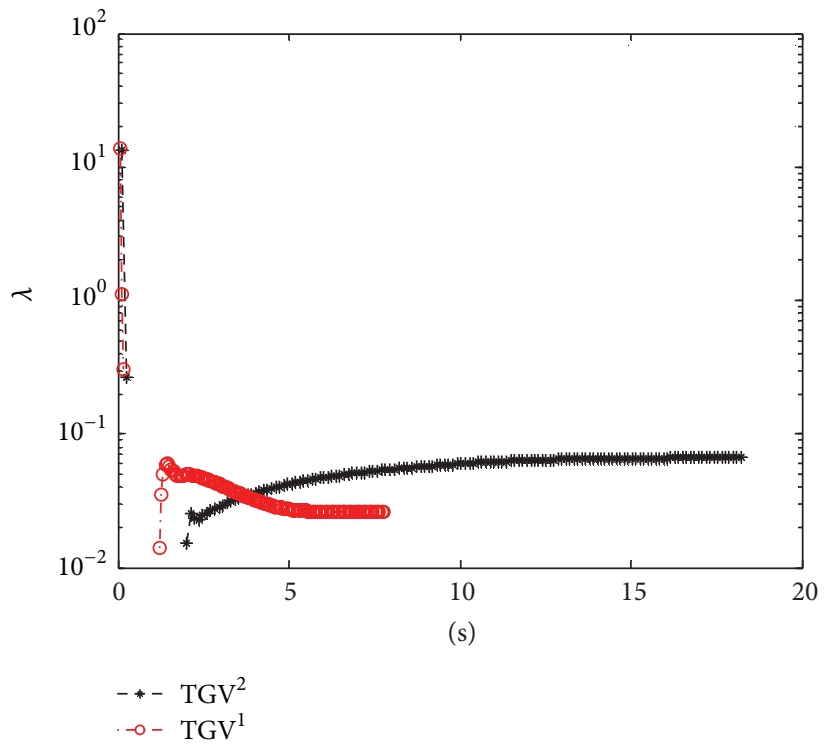

(a)

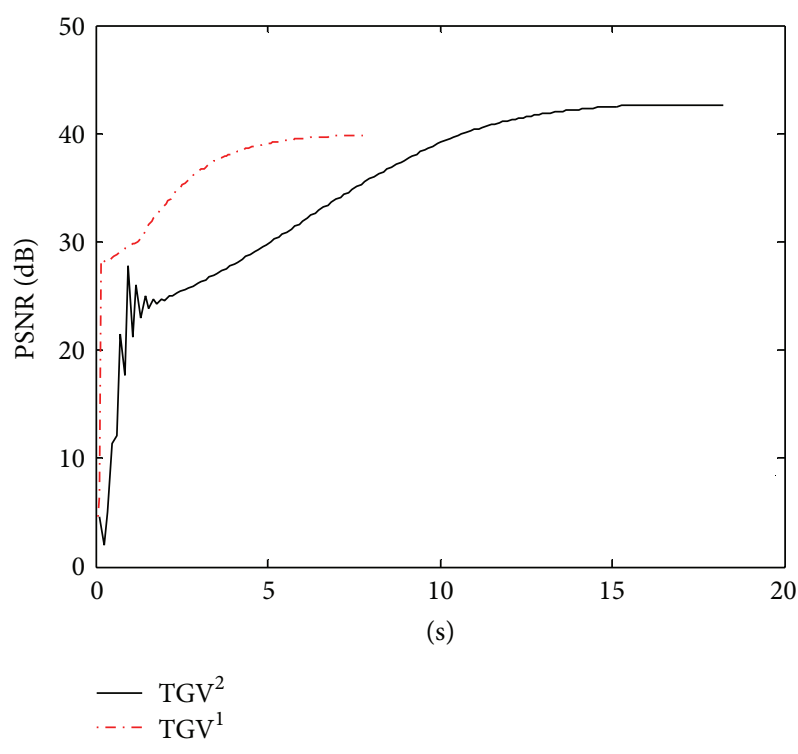

(b)

FIGURE 2: Evolutions of $\lambda_{s}(a)$ and PSNRs (b) achieved by TGV ${ }^{2}$ ID-ADMM (TGV ${ }^{2}$ ) and TGV ID-ADMM $\left(\mathrm{TGV}^{1}\right)$.

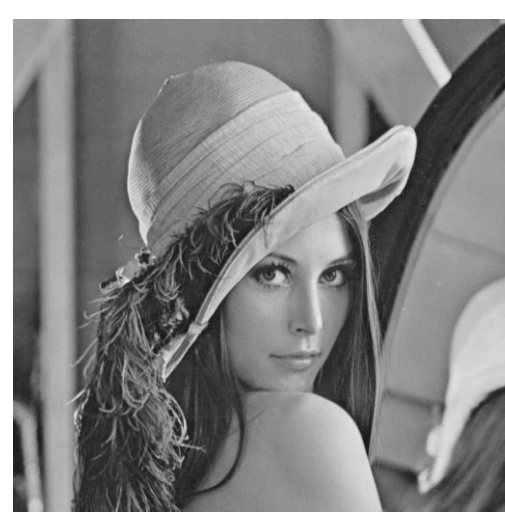

(a)

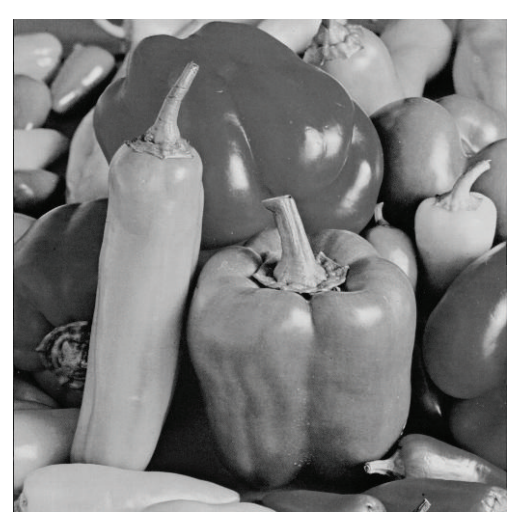

(b)

FIgURE 3: Test images: Lena and Peppers. 


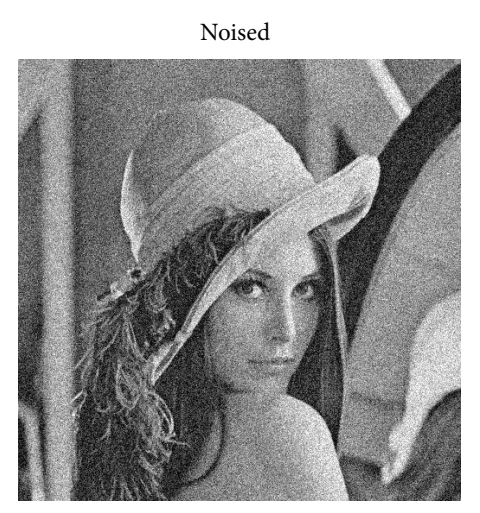

(a)

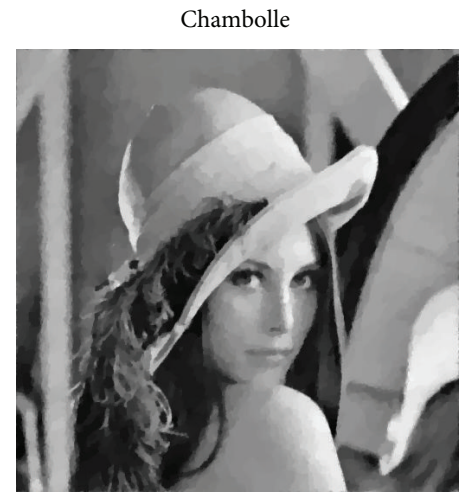

(c)

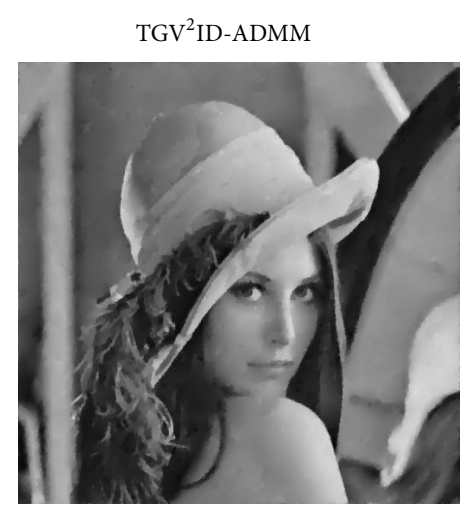

(b)

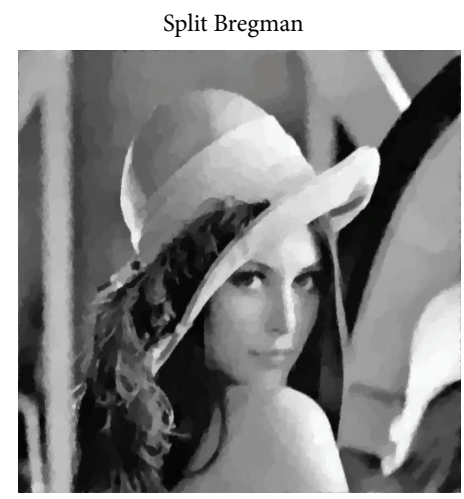

(d)

FIGURE 4: Noised Lena under Gaussian noise of $\sigma=30$ and the restorations by TGV ${ }^{2}$ ID-ADMM, Chambolle, and Split Bregman, respectively.

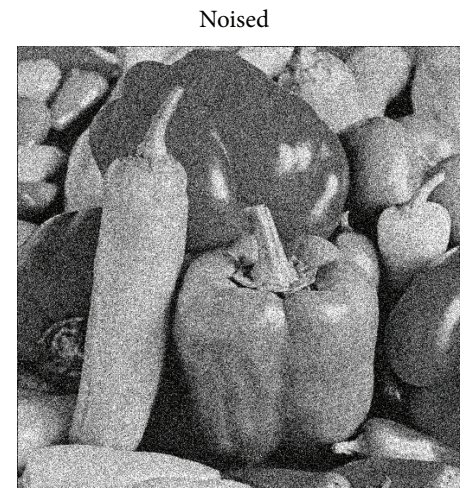

(a)

Chambolle

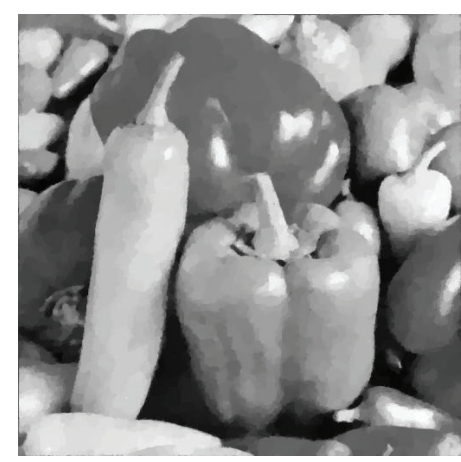

(c)

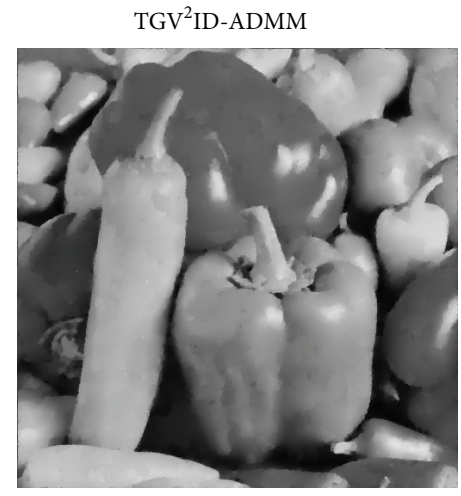

(b)

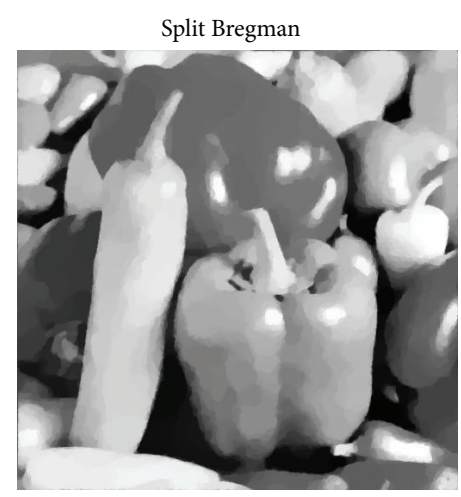

(d)

FIgure 5: Noised Peppers under Gaussian noise of $\sigma=40$ and the restorations by TGV ${ }^{2}$ ID-ADMM, Chambolle, and Split Bregman, respectively. 


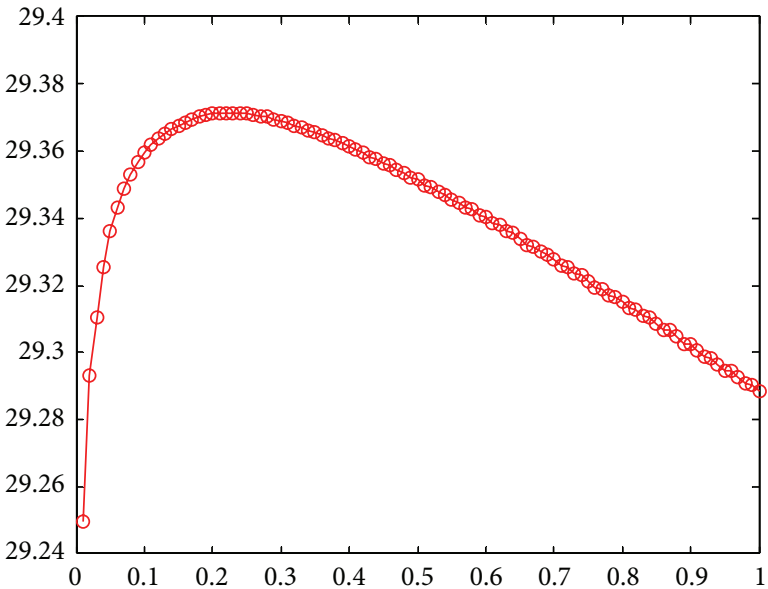

(a) Lena image under Gaussian noise of $\sigma=30$

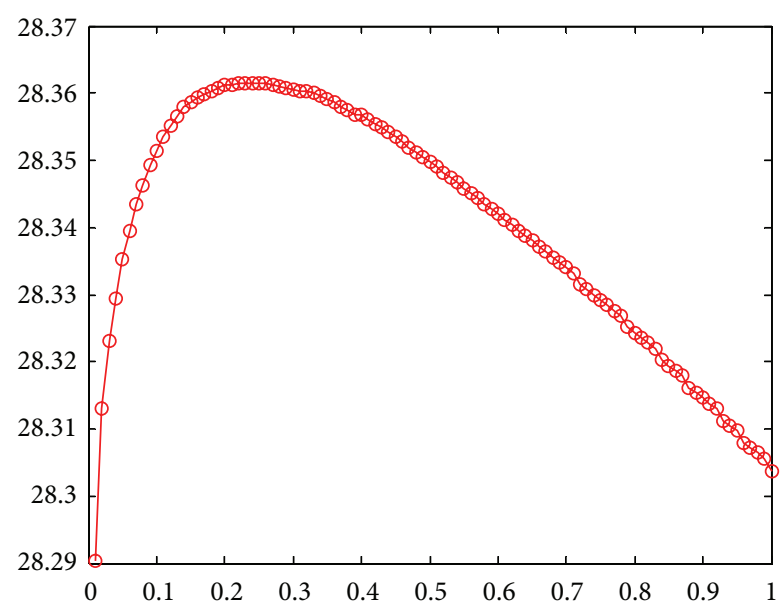

(b) Peppers image under Gaussian noise of $\sigma=40$

Figure 6: PSNR versus $\beta$ for (a) Lena image under Gaussian noise of $\sigma=30$ and (b) Peppers image under Gaussian noise of $\sigma=40$, respectively. The absolute error between the maximum and the minimum of PSNR for each image is less than $0.18 \mathrm{~dB}$.

location is robust towards the variation of image and noise level. The results of our method possess sufficient robustness with respect to the variation of penalty parameters to a certain extent, since the absolute error between the maximum and the minimum of PSNR is less than $0.18 \mathrm{~dB}$ in the experiment, and this error could not introduce obvious distinction in visual quality. In the former two experiments, the setting of $\beta=0.3$ is approximately optimal for the proposed algorithm.

\section{Concluding Remarks}

We propose an adaptive TGV-based model for noise removal in this paper. The variable splitting (VS) and the classical augmented Lagrangian method are used to handle the proposed model. From the experimental results, we observe that the proposed algorithm is effective in suppressing staircasing effect and preserving edges in images, and it is superior to some other famed adaptive denoising methods both in quantitative and in qualitative assessment. Besides, our work can be smoothly generalized to image deblurring problems.

\section{Appendix}

\section{The Equivalent Definition of $\mathrm{TGV}_{\alpha}^{2}$}

In discrete version, we have

$$
\begin{aligned}
& \operatorname{TGV}_{\boldsymbol{\alpha}}^{2}(\mathbf{u})=\max _{\mathbf{v}, \mathbf{d}}\{\langle\mathbf{u}, \operatorname{div} \mathbf{d}\rangle \mid \operatorname{div} \mathbf{v}=\mathbf{d}, \\
&\left.\|\mathbf{v}\|_{\infty} \leq \alpha_{0},\|\mathbf{d}\|_{\infty} \leq \alpha_{1}\right\},
\end{aligned}
$$

where

$$
\begin{gathered}
\|\mathbf{v}\|_{\infty}=\max _{i, j}\left(v_{i, j, 1}^{2}+v_{i, j, 2}^{2}+2 v_{i, j, 3}^{2}\right)^{1 / 2}, \\
\|\mathbf{d}\|_{\infty}=\max _{i, j}\left(d_{i, j, 1}^{2}+d_{i, j, 2}^{2}\right)^{1 / 2} \\
\left(\mathbf{v}_{i, j}=\left[\begin{array}{ll}
v_{i, j, 1} & v_{i, j, 3} \\
v_{i, j, 3} & v_{i, j, 2}
\end{array}\right], \mathbf{d}_{i, j}=\left[d_{i, j, 1}, d_{i, j, 2}\right]\right) .
\end{gathered}
$$

Therefore, according to the Lagrange duality, we have

$$
\begin{aligned}
\operatorname{TGV}_{\boldsymbol{\alpha}}^{2}(\mathbf{u}) & =\min _{\mathbf{p}} \max _{\|\mathbf{v}\|_{\infty} \leq \alpha_{0},\|\mathbf{d}\|_{\infty} \leq \alpha_{1}}\langle\mathbf{u}, \operatorname{div} \mathbf{d}\rangle+\langle\mathbf{p}, \mathbf{d}-\operatorname{div} \mathbf{v}\rangle \\
& =\min _{\mathbf{p}} \max _{\|\mathbf{v}\|_{\infty} \leq \alpha_{0},\|\mathbf{d}\|_{\infty} \leq \alpha_{1}}\langle-\nabla \mathbf{u}, \mathbf{d}\rangle+\langle\mathbf{p}, \mathbf{d}\rangle+\langle\varepsilon(\mathbf{p}), \mathbf{v}\rangle \\
& =\min _{\mathbf{p}} \max _{\|\mathbf{v}\|_{\infty} \leq \alpha_{0},\|\mathbf{d}\|_{\infty} \leq \alpha_{1}}\langle\mathbf{p}-\nabla \mathbf{u}, \mathbf{d}\rangle+\langle\varepsilon(\mathbf{p}), \mathbf{v}\rangle \\
& =\min _{\mathbf{p}} \alpha_{0}\|\varepsilon(\mathbf{p})\|_{1}+\alpha_{1}\|\nabla \mathbf{u}-\mathbf{p}\|_{1} .
\end{aligned}
$$

\section{Conflict of Interests}

The authors declare that there is no conflict of interests regarding the publication of this paper.

\section{Acknowledgments}

The authors would like to thank Editor Fatih Yaman and anonymous referees for their valuable comments. Their help has greatly enhanced the quality of this paper. This work was partially supported by the National Natural Science Foundation of China under Grant nos. 61203189, 61104223, and 
61374120 and the National Science Fund for Distinguished Young Scholars of China under Grant no. 61025014.

\section{References}

[1] L. I. Rudin, S. Osher, and E. Fatemi, "Nonlinear total variation based noise removal algorithms," Physica D: Nonlinear Phenomena, vol. 60, no. 1-4, pp. 259-268, 1992.

[2] T. Chan, S. Esedoglu, F. Park, and A. Yip, "Recent developments in total variation image restoration," in Mathematical Models of Computer Vision, Springer, New York, NY, USA, 2005.

[3] Y. L. You and M. Kaveh, "Fourth-order partial differential equations for noise removal," IEEE Transactions on Image Processing, vol. 9, no. 10, pp. 1723-1730, 2000.

[4] M. Lysaker, A. Lundervold, and X.-C. Tai, "Noise removal using fourth-order partial differential equation with applications to medical magnetic resonance images in space and time," IEEE Transactions on Image Processing, vol. 12, no. 12, pp. 1579-1589, 2003.

[5] T. F. Chan, S. Esedoglu, and F. Park, "A fourth order dual method for staircase reduction in texture extraction and image restoration problems," in Proceedings of the 17th IEEE International Conference on Image Processing (ICIP '10), pp. 4137-4140, Los Angeles, Calif, USA, September 2010.

[6] M. R. Hajiaboli, "An anisotropic fourth-order diffusion filter for image noise removal," International Journal of Computer Vision, vol. 92, no. 2, pp. 177-191, 2011.

[7] T. Liu and Z. Xiang, "Image restoration combining the secondorder and fourth-order PDEs," Mathematical Problems in Engineering, vol. 2013, Article ID 743891, 7 pages, 2013.

[8] Y. Wang, J. Yang, W. Yin, and Y. Zhang, "A new alternating minimization algorithm for total variation image reconstruction," SIAM Journal on Imaging Sciences, vol. 1, no. 3, pp. 248-272, 2008.

[9] N. B. Brás, J. Bioucas-Dias, R. C. Martins, and A. C. Serra, "An alternating direction algorithm for total variation reconstruction of distributed parameters," IEEE Transactions on Image Processing, vol. 21, no. 6, pp. 3004-3016, 2012.

[10] C. He, C. Hu, W. Zhang, B. Shi, and X. Hu, "Fast total-variation image deconvolution with adaptive parameter estimation via split Bregman method," Mathematical Problems in Engineering, vol. 2014, Article ID 617026, 9 pages, 2014.

[11] T. Goldstein, B. O'Donoghue, S. Setzer, and R. Baraniuk, "Fast alternating direction optimization methods," UCLA CAM Report, 2012.

[12] T. Chan, A. Marquina, and P. Mulet, "High-order total variation-based image restoration," SIAM Journal on Scientific Computing, vol. 22, no. 2, pp. 503-516, 2001.

[13] O. Scherzer, "Denoising with higher order derivatives of bounded variation and an application to parameter estimation," Computing, vol. 60, no. 1, pp. 1-27, 1998.

[14] A. Chambolle and P.-L. Lions, "Image recovery via total variation minimization and related problems," Numerische Mathematik, vol. 76, no. 2, pp. 167-188, 1997.

[15] G. Dal Maso, I. Fonseca, G. Leoni, and M. Morini, "A higher order model for image restoration: the one-dimensional case," SIAM Journal on Mathematical Analysis, vol. 40, pp. 2351-2391, 2009.

[16] B. Shi, Z. F. Pang, and Y. F. Yang, "Image restoration based on the hybrid total-variation-type model," Abstract and Applied Analysis, vol. 2012, Article ID 376802, 30 pages, 2012.
[17] K. Bredies, K. Kunisch, and T. Pock, “Total generalized variation," SIAM Journal on Imaging Sciences, vol. 3, no. 3, pp. 492526, 2010.

[18] Yu. Nesterov, "A method for solving a convex programming problem with convergence rate $\mathrm{O}(1 / \mathrm{k} 2)$," Soviet Mathematics Doklady, vol. 27, pp. 372-376, 1983.

[19] R. Glowinski and P. Le Tallec, Augmented Lagrangians and Operator-Splitting Methods in Nonlinear Mechanics, Studies in Applied Mathematics 9, SIAM, Philadelphia, Pa, USA, 1989.

[20] S. Xie and S. Rahardja, "Alternating direction method for balanced image restoration," IEEE Transactions on Image Processing, vol. 21, no. 11, pp. 4557-4567, 2012.

[21] W. Deng and W. Yin, "On the global and linear convergence of the generalized alternating direction method of multipliers," UCLA CAM Report Cam 12-52, 2012.

[22] W. Guo, J. Qin, and W. Yin, "A new detail-preserving regularity scheme," UCLA CAM Report cam13-04, 2013.

[23] J. Duran, B. Coll, and C. Sbert, "Chambolle's pojection algorithm for total variation denoising," Image Processing on Line, vol. 3, pp. 311-331, 2013.

[24] P. Getreuer, "Rudin-Osher-Fatemi total variation denoising using split Bregman," Image Processing on Line, vol. 2, pp. 7495, 2012.

[25] C. Wu and X.-C. Tai, "Augmented lagrangian method, dual methods, and split Bregman iteration for ROF, vectorial TV, and high order models," SIAM Journal on Imaging Sciences, vol. 3, no. 3, pp. 300-339, 2010. 


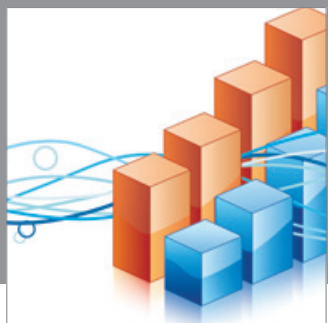

Advances in

Operations Research

mansans

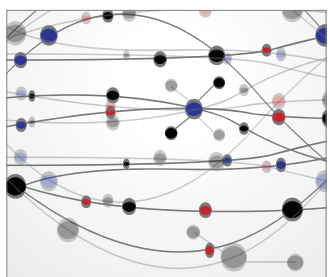

The Scientific World Journal
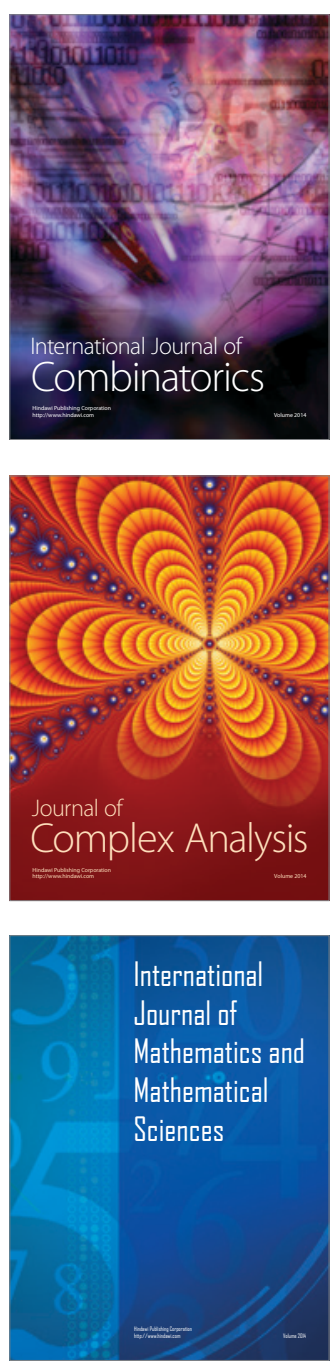
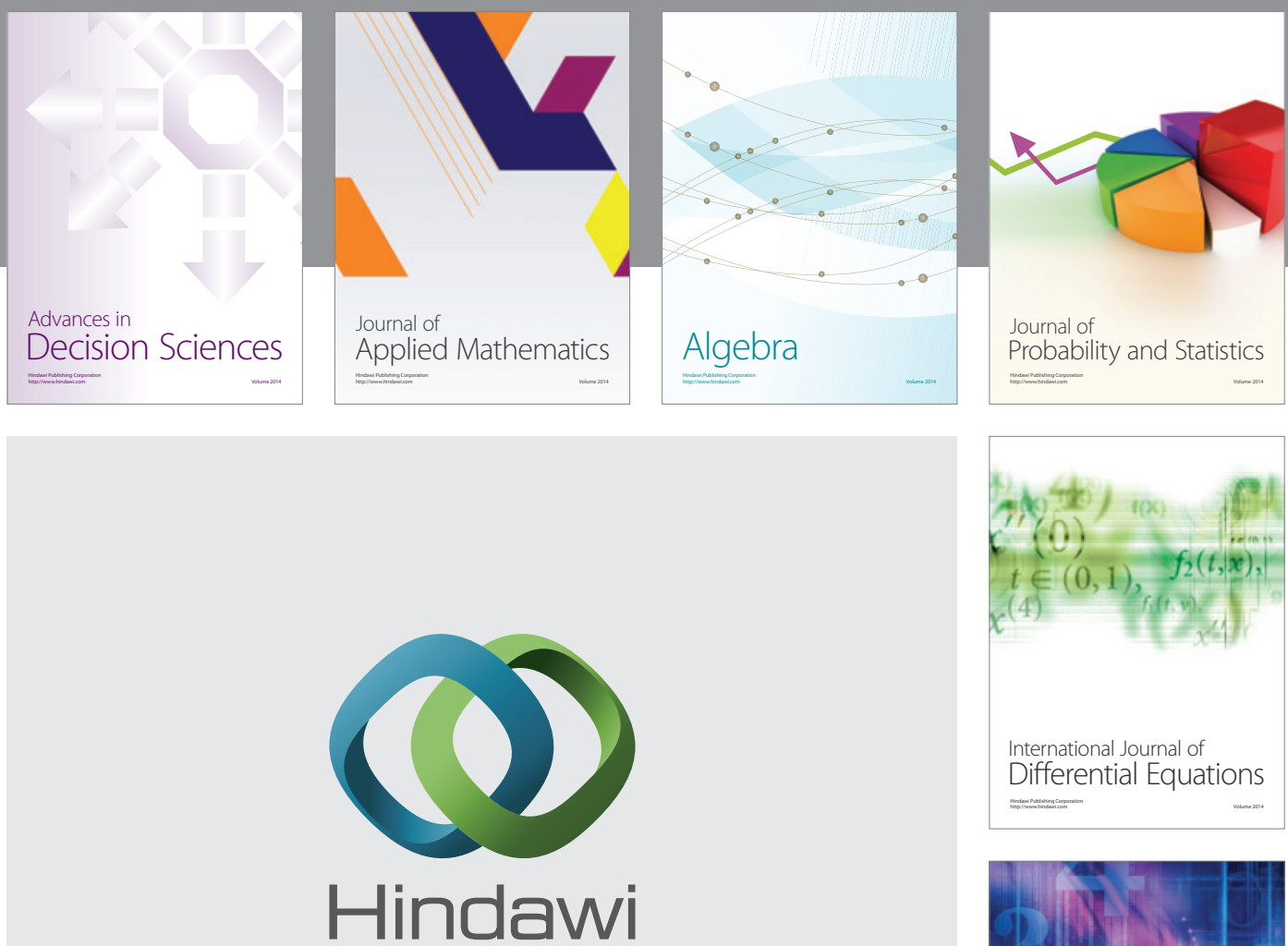

Submit your manuscripts at http://www.hindawi.com
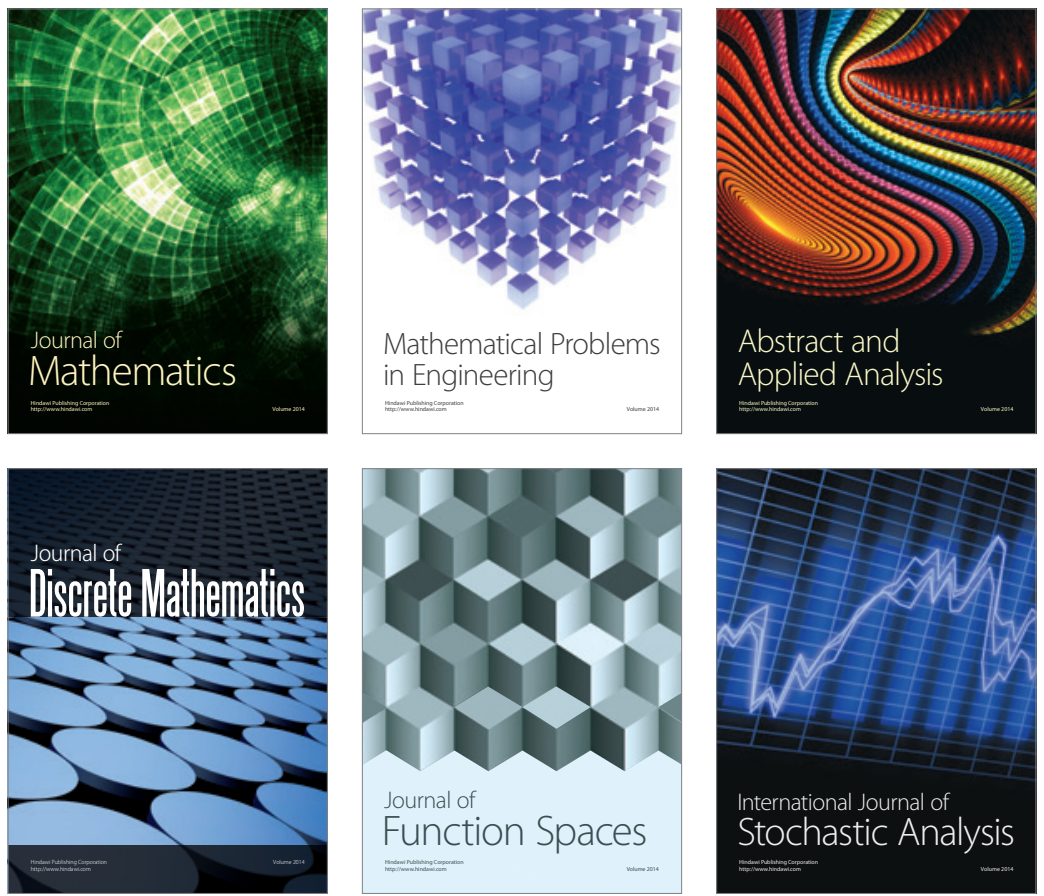

Journal of

Function Spaces

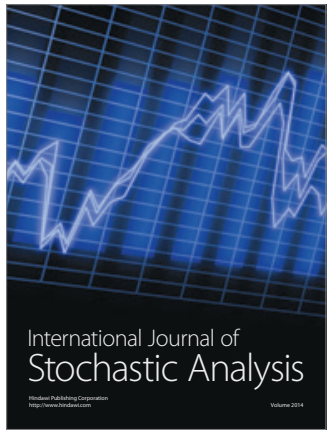

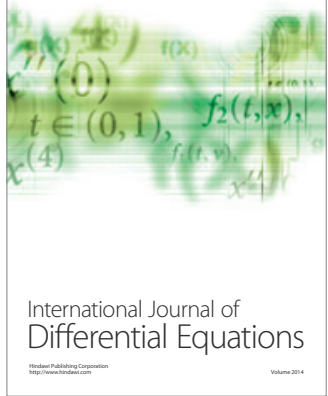
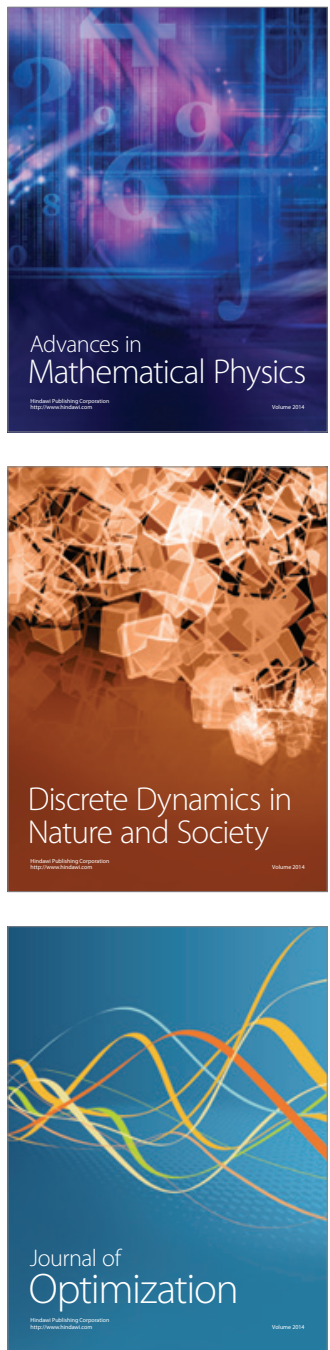